\title{
COHERENT STATES IN BERNOULLI NOISE FUNCTIONALS
}

\author{
CAISHI WANG ${ }^{凶}$ and QI HAN
}

(Received 13 November 2010)

\begin{abstract}
Let $(\Omega, \mathcal{F}, \mathbb{P})$ be a probability space and $Z=\left(Z_{K}\right)_{k \in \mathbb{N}}$ a Bernoulli noise on $(\Omega, \mathcal{F}, \mathbb{P})$ which has the chaotic representation property. In this paper, we investigate a special family of functionals of $Z$, which we call the coherent states. First, with the help of $Z$, we construct a mapping $\phi$ from $l^{2}(\mathbb{N})$ to $\mathcal{L}^{2}(\Omega, \mathcal{F}, \mathbb{P})$ which is called the coherent mapping. We prove that $\phi$ has the continuity property and other properties of operation. We then define functionals of the form $\phi(f)$ with $f \in l^{2}(\mathbb{N})$ as the coherent states and prove that all the coherent states are total in $\mathcal{L}^{2}(\Omega, \mathcal{F}, \mathbb{P})$. We also show that $\phi$ can be used to factorize $\mathcal{L}^{2}(\Omega, \mathcal{F}, \mathbb{P})$. Finally we give an application of the coherent states to calculus of quantum Bernoulli noise.
\end{abstract}

2010 Mathematics subject classification: primary 60H40; secondary 46E30.

Keywords and phrases: Bernoulli noise, coherent state, factorization of functional space.

\section{Introduction}

Bernoulli noise functionals play an important role in many problems such as logarithmic Sobolev inequalities, deviation inequalities and option hedging in mathematical finance (see, for example, [3] and references therein).

In recent years, there has been much interest in Bernoulli noise functionals. In 2001 Émery [1] considered the chaotic representation property of a class of discrete-time stochastic processes including discrete-time Bernoulli noises. Years later, Privault [3] surveyed the discrete-time chaotic calculus, which is a Malliavin-type theory of stochastic calculus for Bernoulli noise functionals. Recently Wang et al. [4] introduced a notion of quantum Bernoulli noises and defined corresponding quantum stochastic integrals, which are actually about operator processes acting on Bernoulli noise functionals. More recently Wang et al. [5] have presented an alternative approach to the discrete-time chaotic calculus.

The authors are supported by National Natural Science Foundation of China (Grant No. 11061032) and Natural Science Foundation of Gansu Province (Grant No. 0710RJZA106). The first author is also partly supported by a grant from Northwest Normal University (Grant No. NWNU-KJCXGC-03-61).

(C) 2011 Australian Mathematical Publishing Association Inc. 0004-9727/2011 \$16.00 
Let $(\Omega, \mathcal{F}, \mathbb{P})$ be a probability space and $Z=\left(Z_{K}\right)_{k \in \mathbb{N}}$ an independent sequence of Bernoulli random variables on $(\Omega, \mathcal{F}, \mathbb{P})$ which has the chaotic representation property [1]. Naturally $Z$ can be viewed as a Bernoulli noise (in discrete time) and random variables on $(\Omega, \mathcal{F}, \mathbb{P})$ can be interpreted as functionals of $Z$.

In this paper we investigate a special family of functionals of $Z$ which we call the coherent states. First, with the help of $Z$, we construct a mapping $\phi$ from $l^{2}(\mathbb{N})$ into $\mathcal{L}^{2}(\Omega, \mathcal{F}, \mathbb{P})$ which is called the coherent mapping. We prove that $\phi$ has the continuity property and other good properties of operation. We then define functionals of the form $\phi(f)$ with $f \in l^{2}(\mathbb{N})$ as the coherent states and prove that all the coherent states are total in $\mathcal{L}^{2}(\Omega, \mathcal{F}, \mathbb{P})$. We also show that $\phi$ can be used to factorize $\mathcal{L}^{2}(\Omega, \mathcal{F}, \mathbb{P})$. Finally, we give an application of the coherent states to calculus of quantum Bernoulli noise.

Notation and conventions. Let $\mathbb{N}$ be the set of all nonnegative integers. We denote by $l^{2}(\mathbb{N})$ the usual space of square summable real-valued functions on $\mathbb{N}$.

For a subset $S \subset \mathbb{N}$, we define $\Gamma(S)$ as the finite power set of $S$, namely

$$
\Gamma(S)=\{\sigma \mid \sigma \subset S \text { and } \# \sigma<\infty\},
$$

where $\# \sigma$ means the cardinality of $\sigma$ as a set. If $S=\{0,1, \ldots, k\}$, then we simply write $\Gamma_{k]}=\Gamma(S)$. We set $\Gamma_{-1]}=\Gamma(\emptyset)$.

In the following, we write $\Gamma=\Gamma(\mathbb{N})$ for brevity ( $\Gamma$ is clearly countable) and set

$$
\Gamma^{(n)}=\{\sigma \in \Gamma \mid \# \sigma=n\}
$$

for $n \in \mathbb{N}$. By convention, $l^{2}(\Gamma)$ denotes the space of square summable real-valued functions on $\Gamma$. For a function on $\mathbb{N}$, we can define a function $\mathcal{E}_{f}$ on $\Gamma$ as

$$
\mathcal{E}_{f}(\sigma)=\prod_{k \in \sigma} f(k), \quad \sigma \in \Gamma,
$$

where $\mathcal{E}_{f}(\emptyset)=1$. It can be shown that $\mathcal{E}_{f} \in l^{2}(\Gamma)$ whenever $f \in l^{2}(\mathbb{N})$.

\section{Bernoulli noise}

We assume throughout that $(\Omega, \mathcal{F}, \mathbb{P})$ is a probability space and $Z=\left(Z_{k}\right)_{k \in \mathbb{N}}$ is an independent sequence of random variables on $(\Omega, \mathcal{F}, \mathbb{P})$ which satisfies

$$
\mathbb{P}\left\{Z_{k}=a_{k}\right\}=p_{k}, \quad \mathbb{P}\left\{Z_{k}=-1 / a_{k}\right\}=q_{k}, \quad k \in \mathbb{N},
$$

with $a_{k}=\sqrt{q_{k} / p_{k}}, q_{k}=1-p_{k}$ and $0<p_{k}<1$ and, moreover, $\mathcal{F}$ is generated by the sequence $\left(Z_{k}\right)_{k \in \mathbb{N}}$, namely

$$
\mathcal{F}=\sigma\left(Z_{k} ; k \in \mathbb{N}\right) .
$$

Such a sequence of random variables does exist (see, for example, [1, 3]). It can be verified that the sequence $Z=\left(Z_{k}\right)_{k \in \mathbb{N}}$ satisfies the discrete structure equation

$$
Z_{k}^{2}=1+\lambda_{k} Z_{k}, \quad k \in \mathbb{N},
$$

with $\lambda_{k}=\left(1-2 p_{k}\right) / \sqrt{p_{k}\left(1-p_{k}\right)}$. 
In the following, we set $\mathcal{F}_{-1}=\{\varnothing, \Omega\}$ and, for $k \in \mathbb{N}$, we denote by $\mathcal{F}_{k}$ the $\sigma$-field generated by $\left(Z_{j}\right)_{0 \leq j \leq k}$, namely

$$
\mathcal{F}_{k}=\sigma\left(Z_{j} ; 0 \leq j \leq k\right)
$$

In this way, the sequence $\left(\mathcal{F}_{k}\right)_{k \geq-1}$ forms a filtration of $\sigma$-fields over $(\Omega, \mathcal{F}, \mathbb{P})$. By convention, we use $\mathbb{E}$ to mean the expectation operator with respect to $\mathbb{P}$.

REMARK 2.1. As will be seen seen, $Z$ is actually a discrete-time Bernoulli stochastic process. And if we put

$$
M_{n}=\sum_{k=0}^{n} Z_{k}, \quad n \in \mathbb{N},
$$

then $\left(M_{n}\right)_{n \in \mathbb{N}}$ is a martingale. Hence $Z$ can be viewed as a (discrete-time) Bernoulli noise. Owing to the relation described by (2.2), random variables on $(\Omega, \mathcal{F}, \mathbb{P})$ can also be interpreted as functionals of $Z$.

In the following, we always write $\mathcal{L}^{2}(\Omega)=\mathcal{L}^{2}(\Omega, \mathcal{F}, \mathbb{P})$, the space of square integrable random variables on $(\Omega, \mathcal{F}, \mathbb{P})$. The inner product and norm of $\mathcal{L}^{2}(\Omega)$ are denoted $\langle\cdot, \cdot\rangle_{\mathcal{L}^{2}(\Omega)}$ and $\|\cdot\|_{\mathcal{L}^{2}(\Omega)}$, respectively.

Clearly $\left\{Z_{k} \mid k \in \mathbb{N}\right\} \subset \mathcal{L}^{2}(\Omega)$. The next lemma shows that $\left\{Z_{k} \mid k \in \mathbb{N}\right\}$ also has the chaotic representation property (see, for example, [4] for a proof).

LEMMA 2.2. Let $Z_{\emptyset}=1$ and

$$
Z_{\sigma}=\prod_{k \in \sigma} Z_{k}, \quad \sigma \in \Gamma, \sigma \neq \emptyset .
$$

Then the set $\left\{Z_{\sigma} \mid \sigma \in \Gamma\right\}$ forms a countable orthonormal basis for $\mathcal{L}^{2}(\Omega)$.

It is known that $l^{2}(\Gamma)$ has an orthonormal basis $\left\{\delta_{\sigma} \mid \sigma \in \Gamma\right\}$, where $\delta_{\sigma}$ is the Dirac delta function on $\Gamma$. Hence we come to the next lemma.

LEMMA 2.3. There exists a unique isometric isomorphism $\mathbb{J}: l^{2}(\Gamma) \mapsto \mathcal{L}^{2}(\Omega)$ such that

$$
\mathbb{J}(f)=\sum_{\sigma \in \Gamma} f(\sigma) Z_{\sigma}, \quad f \in l^{2}(\Gamma)
$$

where the series converges in the norm of $\mathcal{L}^{2}(\Omega)$.

Definition 2.4 [5]. The isometric isomorphism $\mathbb{J}$ stated in Lemma 2.3 is referred to as the full Wiener integral operator with respect to $Z$.

\section{Coherent mapping}

In the present section we will define a continuous mapping $\phi$ from $l^{2}(\mathbb{N})$ to $\mathcal{L}^{2}(\Omega)$, which is called the coherent mapping. We will also show basic properties of $\phi$. 
THEOREM 3.1. Let $f \in l^{2}(\mathbb{N})$. Then the following infinite product of random variables converges in $\mathcal{L}^{2}(\Omega)$ :

$$
\prod_{k=0}^{\infty}\left(1+f(k) Z_{k}\right) .
$$

PROOF. First we note that $\prod_{k=0}^{\infty}\left(1+|f(k)|^{2}\right)$ converges as an infinite product of positive numbers since

$$
\sum_{k=0}^{\infty}|f(k)|^{2}<\infty
$$

Now let $\eta_{n}=\prod_{k=0}^{n}\left(1+f(k) Z_{k}\right), n \geq 0$. Then $\eta_{n} \in \mathcal{L}^{2}(\Omega), n \geq 0$. And for any $m$, $n \geq 0$ with $m<n$, by the independence of the sequence $\left(Z_{k}\right)_{k \in \mathbb{N}}$,

$$
\begin{aligned}
\left\|\eta_{n}-\eta_{m}\right\|_{\mathcal{L}^{2}(\Omega)}^{2}=\mathbb{E} & {\left[\prod_{k=0}^{n}\left(1+f(k) Z_{k}\right)^{2}+\prod_{k=0}^{m}\left(1+f(k) Z_{k}\right)^{2}\right.} \\
& \left.\quad-2 \prod_{k=0}^{m}\left(1+f(k) Z_{k}\right)^{2} \prod_{k=m+1}^{n}\left(1+f(k) Z_{k}\right)\right] \\
= & \prod_{k=0}^{n} \mathbb{E}\left(1+f(k) Z_{k}\right)^{2}+\prod_{k=0}^{m} \mathbb{E}\left(1+f(k) Z_{k}\right)^{2} \\
& \quad-2 \prod_{k=0}^{m} \mathbb{E}\left(1+f(k) Z_{k}\right)^{2} \prod_{k=m+1}^{n} \mathbb{E}\left(1+f(k) Z_{k}\right) \\
= & \prod_{k=0}^{n}\left(1+|f(k)|^{2}\right)-\prod_{k=0}^{m}\left(1+|f(k)|^{2}\right) .
\end{aligned}
$$

Thus the sequence $\eta_{n}, n \geq 0$, converges in $\mathcal{L}^{2}(\Omega)$, that is, (3.1) converges in $\mathcal{L}^{2}(\Omega)$.

Definition 3.2. The coherent mapping $\phi$ is the one from $l^{2}(\mathbb{N})$ to $\mathcal{L}^{2}(\Omega)$ given by

$$
\phi(f)=\prod_{k=0}^{\infty}\left(1+f(k) Z_{k}\right), \quad f \in l^{2}(\mathbb{N}) .
$$

The next theorem shows some metric properties of the coherent mapping.

Theorem 3.3. Let $f, g \in l^{2}(\mathbb{N})$. Then

$$
\langle\phi(f), \phi(g)\rangle_{\mathcal{L}^{2}(\Omega)}=\prod_{k=0}^{\infty}(1+f(k) g(k)) .
$$

In particular,

$$
\|\phi(f)\|_{\mathcal{L}^{2}(\Omega)}^{2}=\prod_{k=0}^{\infty}\left(1+|f(k)|^{2}\right) .
$$


PROOF. We first note that the infinite product on the right-hand side of (3.3) absolutely converges since

$$
\sum_{k=0}^{\infty}|f(k) g(k)|<\infty .
$$

Now for $f, g \in l^{2}(\mathbb{N})$, by the continuity of the inner product as well as the independence of the sequence $\left(Z_{k}\right)_{k \in \mathbb{N}}$, we get

$$
\begin{aligned}
\langle\phi(f), \phi(g)\rangle_{\mathcal{L}^{2}(\Omega)} & =\lim _{n \rightarrow \infty}\left\langle\prod_{k=0}^{n}\left(1+f(k) Z_{k}\right), \prod_{k=0}^{n}\left(1+g(k) Z_{k}\right)\right\rangle_{\mathcal{L}^{2}(\Omega)} \\
& =\lim _{n \rightarrow \infty} \prod_{k=0}^{n} \mathbb{E}\left(1+f(k) Z_{k}+g(k) Z_{k}+f(k) g(k) Z_{k}^{2}\right) \\
& =\prod_{k=0}^{\infty}(1+f(k) g(k)) .
\end{aligned}
$$

This completes the proof.

REMARK 3.4. From the above theorem and inequality $\ln \left(1+x^{2}\right) \leq x^{2}, x \in \mathbb{R}$, it follows that

$$
\|\phi(f)\|_{\mathcal{L}^{2}(\Omega)} \leq \exp \left(\frac{1}{2}\|f\|_{l^{2}(\mathbb{N})}^{2}\right), \quad f \in l^{2}(\mathbb{N}) .
$$

We note that the left-hand side of (3.5) can be strictly less than the right-hand side.

THEOREM 3.5. Let $\Phi$ be the function on $l^{2}(\mathbb{N}) \times l^{2}(\mathbb{N})$ defined by

$$
\Phi(f, g)=\langle\phi(f), \phi(g)\rangle_{\mathcal{L}^{2}(\Omega)}, \quad f, g \in l^{2}(\mathbb{N}) .
$$

Then $\Phi$ is continuous.

PROOF. It follows from (3.3) that

$$
\Phi(f, g)=\prod_{k=0}^{\infty}(1+f(k) g(k)), \quad f, g \in l^{2}(\mathbb{N}) .
$$

Now let $f, g \in l^{2}(\mathbb{N})$ and $f_{n}, g_{n} \in l^{2}(\mathbb{N}), n \geq 1$, be two sequences satisfying

$$
\lim _{n \rightarrow \infty}\left\|f_{n}-f\right\|_{l^{2}(\mathbb{N})}=0, \quad \lim _{n \rightarrow \infty}\left\|g_{n}-g\right\|_{l^{2}(\mathbb{N})}=0 .
$$

We need to show that $\Phi\left(f_{n}, g_{n}\right) \rightarrow \Phi(f, g)$ as $n \rightarrow \infty$.

Since $\sum_{k=0}^{\infty}|f(k) g(k)|<\infty$, we can take $k_{0} \geq 1$ such that $|f(k) g(k)|<1 / 4$ for all $k \geq k_{0}$. We can also take $n_{0} \geq 1$ such that

$$
c\left\|f_{n}-f\right\|_{l^{2}(\mathbb{N})}+\|f\|_{l^{2}(\mathbb{N})}\left\|g_{n}-g\right\|_{l^{2}(\mathbb{N})}<\frac{1}{4}, \quad n \geq n_{0},
$$

where $c=\sup _{n \geq 1}\left\|g_{n}\right\|_{l^{2}(\mathbb{N})}$. Thus for all $n \geq n_{0}$ and all $k \geq k_{0}$,

$$
\left|f_{n}(k) g_{n}(k)\right| \leq \alpha\left\|f_{n}-f\right\|_{l^{2}(\mathbb{N})}+\|f\|_{l^{2}(\mathbb{N})}\left\|g_{n}-g\right\|_{l^{2}(\mathbb{N})}+|f(k) g(k)|<\frac{1}{2} .
$$


It is well known that

$$
|\ln (1+x)-\ln (1+y)| \leq 2|x-y| \text { for all } x, y \in\left[-\frac{1}{2},+\infty\right) .
$$

Thus, for all $j \geq 0$,

$$
\begin{aligned}
& \left|\sum_{k=k_{0}}^{\infty} \ln \left[1+f_{j+n_{0}}(k) g_{j+n_{0}}(k)\right]-\sum_{k=k_{0}}^{\infty} \ln [1+f(k) g(k)]\right| \\
& \quad \leq \sum_{k=k_{0}}^{\infty}\left|\ln \left[1+f_{j+n_{0}}(k) g_{j+n_{0}}(k)\right]-\ln [1+f(k) g(k)]\right| \\
& \quad \leq 2 \sum_{k=k_{0}}^{\infty}\left|f_{j+n_{0}}(k) g_{j+n_{0}}(k)-f(k) g(k)\right| \\
& \leq 2\left\|f_{j+n_{0}}-f\right\|_{l^{2}(\mathbb{N})}\left\|g_{j+n_{0}}\right\|_{l^{2}(\mathbb{N})}+2\|f\|_{l^{2}(\mathbb{N})}\left\|g_{j+n_{0}}-g\right\|_{l^{2}(\mathbb{N})}
\end{aligned}
$$

This implies that

$$
\lim _{j \mapsto \infty} \sum_{k=k_{0}}^{\infty} \ln \left[1+f_{j+n_{0}}(k) g_{j+n_{0}}(k)\right]=\sum_{k=k_{0}}^{\infty} \ln [1+f(k) g(k)] .
$$

On the other hand, for all $j \geq 0$, we see that

$$
\begin{aligned}
\Phi\left(f_{j+n_{0}}, g_{j+n_{0}}\right)= & \prod_{k=0}^{k_{0}-1}\left[1+f_{j+n_{0}}(k) g_{j+n_{0}}(k)\right] \\
& \quad \times \exp \left\{\sum_{k=k_{0}}^{\infty} \ln \left[1+f_{j+n_{0}}(k) g_{j+n_{0}}(k)\right]\right\} .
\end{aligned}
$$

This, together with the continuity of exponential function, implies that

$$
\begin{aligned}
\lim _{j \mapsto \infty} \Phi\left(f_{j+n_{0}}, g_{j+n_{0}}\right)= & \prod_{k=0}^{k_{0}-1}[1+f(k) g(k)] \\
& \quad \times \exp \left\{\sum_{k=k_{0}}^{\infty} \ln [1+f(k) g(k)]\right\}=\Phi(f, g) .
\end{aligned}
$$

Thus $\Phi\left(f_{n}, g_{n}\right) \rightarrow \Phi(f, g)$ as $n \rightarrow \infty$. This completes the proof.

THEOREM 3.6. The coherent mapping $\phi: l^{2}(\mathbb{N}) \mapsto \mathcal{L}^{2}(\Omega)$ is continuous.

PROOF. Let $f \in l^{2}(\mathbb{N})$ and $f_{n} \in l^{2}(\mathbb{N}), n \geq 1$, be a sequence converging to $f$ in the norm. Then

$$
\left\|\phi\left(f_{n}\right)-\phi(f)\right\|_{\mathcal{L}^{2}(\Omega)}^{2}=\Phi\left(f_{n}, f_{n}\right)-2 \Phi\left(f_{n}, f\right)+\Phi(f, f) \quad n \geq 1 .
$$

Thus by Theorem 3.5 we get $\lim _{n \rightarrow \infty}\left\|\phi\left(f_{n}\right)-\phi(f)\right\|_{\mathcal{L}^{2}(\Omega)}=0$. This verifies the continuity of $\phi$. 
Recall that if $f \in l^{2}(\mathbb{N})$, then $\mathcal{E}_{f} \in l^{2}(\Gamma)$ (see (1.3) for the definition of $\mathcal{E}_{f}$ ). The next theorem shows the relationship between $\phi(f)$ and $\mathcal{E}_{f}$.

THEOREM 3.7. Let $f \in l^{2}(\mathbb{N})$. Then $\mathbb{J}\left(\mathcal{E}_{f}\right)=\phi(f)$, where $\mathbb{J}$ is the full Wiener integral operator.

PROOF. For each $n \geq 0$,

$$
\prod_{k=0}^{n}\left(1+f(k) Z_{k}\right)=\sum_{\sigma \in \Gamma_{n]}} \mathcal{E}_{f}(\sigma) Z_{\sigma} .
$$

Then, by letting $n \rightarrow \infty$, we get

$$
\prod_{k=0}^{\infty}\left(1+f(k) Z_{k}\right)=\sum_{\sigma \in \Gamma} \mathcal{E}_{f}(\sigma) Z_{\sigma}
$$

that is, $\phi(f)=\mathbb{J}\left(\mathcal{E}_{f}\right)$.

For $S \subset \mathbb{N}$ with $S \neq \emptyset$, we define $\mathcal{F}_{S}$ as the $\sigma$-field generated by $\left(Z_{k}\right)_{k \in S}$ :

$$
\mathcal{F}_{S}=\sigma\left(Z_{k} ; k \in S\right) \text {. }
$$

As usual, $\mathbb{E}\left[\cdot \mid \mathcal{F}_{S}\right]$ denotes the conditional expectation given $\mathcal{F}_{S}$. The next theorem shows an interesting link between the coherent mapping and the conditional expectation operator.

TheOREM 3.8. Let $S \subset \mathbb{N}$ with $S \neq \emptyset$. Then

$$
\mathbb{E}\left[\phi(f) \mid \mathcal{F}_{S}\right]=\phi\left(f \mathbf{1}_{S}\right), \quad f \in l^{2}(\mathbb{N}),
$$

where $\mathbf{1}_{S}$ stands for the indicator of $S$.

PROOF. For $f \in l^{2}(\mathbb{N})$, we can show that

$$
\phi(f)=\prod_{k \in S}\left(1+f(k) Z_{k}\right) \prod_{k \in \mathbb{N} \backslash S}\left(1+f(k) Z_{k}\right),
$$

which, together with the independence of the sequence $\left(Z_{k}\right)_{k \in \mathbb{N}}$, implies (3.8).

\section{Coherent states}

In this section, we will show the totality of the set $\left\{\phi(f) \mid f \in l^{2}(\mathbb{N})\right\}$ in $\mathcal{L}^{2}(\Omega)$ and other related results. We first make some preparations. Let $\mathfrak{F}$ be the symmetric Fock space over $l^{2}(\mathbb{N})$, namely

$$
\mathfrak{F}=\bigoplus_{n=0}^{\infty} l_{s}^{2}\left(\mathbb{N}^{n}\right)
$$

where $l_{s}^{2}\left(\mathbb{N}^{0}\right)=\mathbb{R}$ and $l_{s}^{2}\left(\mathbb{N}^{n}\right)=\left\{F \in l^{2}\left(\mathbb{N}^{n}\right) \mid F\right.$ is symmetric $\}$ for $n \geq 1$. It is well known that $\left\{e(f) \mid f \in l^{2}(\mathbb{N})\right\}$ is a total subset of $\mathfrak{F}$, where $e(f)$ is the exponential 
vector defined by

$$
e(f)=\bigoplus_{n=0}^{\infty} \frac{1}{\sqrt{n !}} f^{\otimes n}
$$

See, for example, [2] for general theory of Fock space.

DEFINITION 4.1. For $f \in l^{2}(\mathbb{N})$, we call $\phi(f)$ the coherent state corresponding to $f$, where $\phi$ is the coherent mapping (see Definition 3.2).

THEOREM 4.2. The coherent state set $\left\{\phi(f) \mid f \in l^{2}(\mathbb{N})\right\}$ is total in $\mathcal{L}^{2}(\Omega)$.

Proof. Let $\xi \in\left\{\phi(f) \mid f \in l^{2}(\mathbb{N})\right\}^{\perp}$. Then there exists $F \in l^{2}(\Gamma)$ such that $\xi=$ $\mathbb{J}(F)$, where $\mathbb{I}$ is the full Wiener integral operator (see (2.6) for its definition).

Define $\tilde{F}_{0}=F(\emptyset)$ and for $n \geq 1$ define a function $\tilde{F}_{n}$ on $\mathbb{N}^{n}$ as

$$
\tilde{F}_{n}\left(k_{1}, k_{2}, \ldots, k_{n}\right)= \begin{cases}\frac{1}{\sqrt{n !}} F\left(\left\{k_{1}, k_{2}, \ldots, k_{n}\right\}\right), & \left(k_{1}, k_{2}, \ldots, k_{n}\right) \in \triangle_{n} ; \\ 0, & \left(k_{1}, k_{2}, \ldots, k_{n}\right) \in \mathbb{N}^{n} \backslash \triangle_{n},\end{cases}
$$

where $\triangle_{n}=\left\{\left(k_{1}, k_{2}, \ldots, k_{n}\right) \in \mathbb{N}^{n} \mid k_{i} \neq k_{j}\right.$ for $\left.i \neq j, 1 \leq i, j \leq n\right\}$. Then we have

$$
\tilde{F}=\bigoplus_{n=0}^{\infty} \tilde{F}_{n} \in \mathfrak{F}
$$

On the other hand, for each $f \in l^{2}(\mathbb{N})$, in view of $\mathbb{J}\left(\mathcal{E}_{f}\right)=\phi(f)$ and $\mathbb{J}(F)=\xi$, we get

$$
\langle e(f), \tilde{F}\rangle_{\mathfrak{F}}=\left\langle\mathcal{E}_{f}, F\right\rangle_{l^{2}(\Gamma)}=\langle\phi(f), \xi\rangle_{\mathcal{L}^{2}(\Omega)}=0 .
$$

This, together with the totality of $\left\{e(f) \mid f \in l^{2}(\mathbb{N})\right\}$ in $\mathfrak{F}$, yields $\tilde{F}=0$, that is,

$$
\tilde{F}_{n}=0, \quad n \geq 0
$$

which implies that $F=0$. Thus $\xi=\mathbb{J}(F)=0$. This completes the proof.

As an immediate consequence of Theorems 3.6 and 4.2, we have the next useful corollary.

Corollary 4.3. If $D$ is a dense subset of $l^{2}(\mathbb{N})$, then the set $\{\phi(f) \mid f \in D\}$ remains total in $\mathcal{L}^{2}(\Omega)$.

Two functions $f, g \in l^{2}(\mathbb{N})$ are called strongly orthogonal if $f(k) g(k)=0$ for all $k \in \mathbb{N}$.

THEOREM 4.4. If $f, g \in l^{2}(\mathbb{N})$ are strongly orthogonal, then

$$
\phi(f+g)=\phi(f) \phi(g) .
$$


PROOF. For each $n \geq 0$, by the strongly orthogonal property of $f$ and $g$,

$$
\prod_{k=0}^{n}\left[1+(f(k)+g(k)) Z_{k}\right]=\prod_{k=0}^{n}\left(1+f(k) Z_{k}\right) \prod_{k=0}^{n}\left(1+g(k) Z_{k}\right) .
$$

Note that the left-hand side of this equality converges to $\phi(f+g)$ in $\mathcal{L}^{2}(\Omega)$, while the right-hand side converges to $\phi(f) \phi(g)$ in $\mathcal{L}^{1}(\Omega)$. Thus, by letting $n \rightarrow \infty$, we get (4.3).

For $k \in \mathbb{N}$, we simply write $\mathbf{1}_{k]}=\mathbf{1}_{[0, k]}$ and, similarly, $\mathbf{1}_{[k}=\mathbf{1}_{[k,+\infty)}$, which are indicators of $[0, k]$ and $[k,+\infty)$, respectively. If $\mathcal{S}_{1}, \mathcal{S}_{2} \subset \mathcal{L}^{2}(\Omega)$ are nonempty, then we define their algebraic product $\mathcal{S}_{1} \mathcal{S}_{2}$ as

$$
\mathcal{S}_{1} \mathcal{S}_{2}=\left\{\xi \eta \mid \xi \in \mathcal{S}_{1}, \eta \in \mathcal{S}_{2}\right\}
$$

which is a subset of $\mathcal{L}^{1}(\Omega)$ in general. However, we have the next result, which follows immediately from Theorem 4.4.

COROLlary 4.5. Let $\mathcal{D}=\left\{\phi(f) \mid f \in l^{2}(\mathbb{N})\right\}$ be the set of all coherent states. Then, for each $k \in \mathbb{N}$,

$$
\mathcal{D}=\mathcal{D}_{k]} \mathcal{D}_{[k+1}
$$

where $\mathcal{D}_{k]}=\left\{\phi\left(f \mathbf{1}_{k]}\right) \mid f \in l^{2}(\mathbb{N})\right\}$ and $\mathcal{D}_{[k+1}=\left\{\phi\left(f \mathbf{1}_{[k+1}\right) \mid f \in l^{2}(\mathbb{N})\right\}$.

THEOREM 4.6. Let $S \subset \mathbb{N}$ with $S \neq \emptyset$. Then $\left\{\phi\left(f \mathbf{1}_{S}\right) \mid f \in l^{2}(\mathbb{N})\right\}$ is a total subset of $\mathcal{L}^{2}\left(\Omega, \mathcal{F}_{S}, \mathbb{P}\right)$, where $\mathbf{1}_{S}$ stands for the indicator of $S$ and $\mathcal{F}_{S}$ is defined by (3.7).

PROOF. By (3.8), we immediately find that

$$
\left\{\phi\left(f \mathbf{1}_{S}\right) \mid f \in l^{2}(\mathbb{N})\right\} \subset \mathcal{L}^{2}\left(\Omega, \mathcal{F}_{S}, \mathbb{P}\right) .
$$

Now let $\xi \in \mathcal{L}^{2}\left(\Omega, \mathcal{F}_{S}, \mathbb{P}\right)$ be such that $\xi \perp\left\{\phi\left(f \mathbf{1}_{S}\right) \mid f \in l^{2}(\mathbb{N})\right\}$. Then for each $f \in l^{2}(\mathbb{N})$, in view of $\xi=\mathbb{E}\left[\xi \mid \mathcal{F}_{S}\right]$,

$$
\langle\xi, \phi(f)\rangle=\left\langle\mathbb{E}\left[\xi \mid \mathcal{F}_{S}\right], \phi(f)\right\rangle=\left\langle\xi, \mathbb{E}\left[\phi(f) \mid \mathcal{F}_{S}\right]\right\rangle=\left\langle\xi, \phi\left(f \mathbf{1}_{S}\right)\right\rangle=0,
$$

where $\langle\cdot, \cdot\rangle$ means $\langle\cdot, \cdot\rangle_{\mathcal{L}^{2}(\Omega)}$. Thus, by the totality of $\left\{\phi(f) \mid f \in l^{2}(\mathbb{N})\right\}$ in $\mathcal{L}^{2}(\Omega)$, we know that $\xi=0$.

\section{Application}

In this final section, we show an application of the coherent states to calculus of quantum Bernoulli noise [4]. We first make some preparations. For $k \in \mathbb{N}$, we write for brevity

$$
\mathcal{H}_{k]}=\mathcal{L}^{2}\left(\Omega, \mathcal{F}_{k]}, \mathbb{P}\right), \quad \mathcal{H}_{[k}=\mathcal{L}^{2}\left(\Omega, \mathcal{F}_{[k}, \mathbb{P}\right),
$$

where $\mathcal{F}_{k]}=\mathcal{F}_{k}$ (see (2.4) for the definition) and $\mathcal{F}_{[k}=\sigma\left(Z_{j} ; k \leq j<\infty\right)$. It is known [4] that the algebraic product $\mathcal{H}_{k]} \mathcal{H}_{[k+1}$ is a dense subset of $\mathcal{L}^{2}(\Omega)$ for each $k \in \mathbb{N}$. According to Theorem 4.6, if $f \in l^{2}(\mathbb{N})$ then $\phi\left(f \mathbf{1}_{k]}\right) \in \mathcal{H}_{k]}$ and $\phi\left(f \mathbf{1}_{[k}\right) \in \mathcal{H}_{[k}$. 
By an operator process we mean a sequence of bounded linear operators on $\mathcal{L}^{2}(\Omega)$. An operator process $L=\left(L_{k}\right)_{k \in \mathbb{N}}$ is called semi-adapted if, for each $k \in \mathbb{N}, L_{k}$ leaves $\mathcal{H}_{k]}$ invariant. A semi-adapted operator process $L=\left(L_{k}\right)_{k \in \mathbb{N}}$ is called adapted if, for each $k \in \mathbb{N}, L_{k}$ further satisfies

$$
L_{k}(\xi \eta)=\left(L_{k} \xi\right) \eta, \quad \xi \in \mathcal{H}_{k]}, \eta \in \mathcal{H}_{[k+1} .
$$

In [4], the authors characterized adapted operator processes in terms of quantum Bernoulli noise. Here, as an application of the coherent states, we present another characterization of adapted operator processes.

THEOREM 5.1. A semi-adapted operator process $L=\left(L_{k}\right)_{k \in \mathbb{N}}$ is adapted if only if, for each $k \in \mathbb{N}, L_{k}$ satisfies

$$
L_{k} \phi(f)=\left[L_{k} \phi\left(f \mathbf{1}_{k]}\right)\right] \phi\left(f \mathbf{1}_{[k+1}\right), \quad f \in l^{2}(\mathbb{N}),
$$

where $P_{k}$ stands for the orthogonal projection onto $\mathcal{H}_{k]}$.

Proof. Obviously, (5.2) implies (5.3). Below we show that (5.3) also implies (5.2).

Let $\mathcal{D}_{k]}=\left\{\phi\left(f \mathbf{1}_{k]}\right) \mid f \in l^{2}(\mathbb{N})\right\}$ and $\mathcal{D}_{[k+1}=\left\{\phi\left(f \mathbf{1}_{[k+1}\right) \mid f \in l^{2}(\mathbb{N})\right\}$. Then, by Theorem 4.6, we know that $\mathcal{D}_{k]}$ and $\mathcal{D}_{[k+1}$ are total in $\mathcal{H}_{k]}$ and $\mathcal{H}_{[k+1}$, respectively. Let $\xi \in \mathcal{H}_{k]}$ and $\eta \in \mathcal{H}_{[k+1}$ be such that

$$
\xi=\sum_{i=1}^{m} s_{i} \phi\left(f_{i} \mathbf{1}_{k]}\right), \quad \eta=\sum_{j=1}^{n} t_{j} \phi\left(g_{j} \mathbf{1}_{[k+1}\right),
$$

where $s_{i}, t_{j}$ are real numbers and $f_{i}, g_{j} \in l^{2}(\mathbb{N})$. Then it follows from Theorem 4.4 that

$$
\xi \eta=\sum_{i=1}^{m} \sum_{j=1}^{n} s_{i} t_{j} \phi\left(f_{i} \mathbf{1}_{k]}+g_{j} \mathbf{1}_{[k+1}\right) .
$$

Thus, by (5.3),

$$
\begin{aligned}
L_{k}(\xi \eta) & =\sum_{i=1}^{m} \sum_{j=1}^{n} s_{i} t_{j}\left[L_{k} \phi\left(f_{i} \mathbf{1}_{k]}\right)\right] \phi\left(g_{j} \mathbf{1}_{[k+1}\right) \\
& =\sum_{i=1}^{m} s_{i} L_{k} \phi\left(f_{i} \mathbf{1}_{k]}\right) \sum_{j=1}^{n} t_{j} \phi\left(g_{j} \mathbf{1}_{[k+1}\right) \\
& =\left(L_{k} \xi\right) \eta .
\end{aligned}
$$

For general $\xi \in \mathcal{H}_{k]}$ and $\eta \in \mathcal{H}_{[k+1}$, by using the usual method of approximation, we can also get $L_{k}(\xi \eta)=\left(L_{k} \xi\right) \eta$. This completes the proof.

\section{References}

[1] M. Émery, 'A discrete approach to the chaotic representation property', in: Séminaire de Probabilités, XXXV, Lecture Notes in Mathematics, 1755 (Springer, Berlin, 2001), pp. 123-138.

[2] K. R. Parthasarathy, An Introduction to Quantum Stochastic Calculus (Birkhäuser, Basel, 1992). 
[3] N. Privault, 'Stochastic analysis of Bernoulli processes', Probab. Surv 5 (2008), 435-483.

[4] C. S. Wang, H. F. Chai and Y. C. Lu, 'Discrete-time quantum Bernoulli noises', J. Math. Phys. 51 (2010), 053528.

[5] C. S. Wang, Y. C. Lu and H. F. Chai, 'An alternative approach to Privault's discrete-time chaotic calculus', J. Math. Anal. Appl. 373 (2011), 643-654.

CAISHI WANG, School of Mathematics and Information Science, Northwest Normal University, Lanzhou, Gansu 730070, PR China e-mail: wangcs@nwnu.edu.cn, cswangnwnu@163.com

QI HAN, School of Mathematics and Information Science, Northwest Normal University, Lanzhou, Gansu 730070, PR China e-mail: hanqi1978@nwnu.edu.cn 